\title{
Nucleolar NOC1 controls protein synthesis and cell competition in Drosophila
}

Francesca Destefanis ${ }^{1^{*}}$, Valeria Manara ${ }^{1^{*}}$, Stefania Santarelli ${ }^{1^{*}}$, Sheri Zola ${ }^{1}$, Marco Brambilla ${ }^{2}$, Giacomo Viola ${ }^{2}$, Paola Maragno ${ }^{1}$, Gabriella Viero ${ }^{3}$, Maria Enrica Pasini ${ }^{2}$, Marianna Penzo ${ }^{5-6}$ and Paola Bellosta ${ }^{1,2,4,7}$

1- Department CIBIO, University of Trento, Via Sommarive 9, 38123 Trento

2- Department of Biosciences, University of Milano, Via Celoria 25, 20133 Milano

3- Institute of Biophysics, CNR, Via Sommarive 18, 38123 Trento

4- Department of Medicine NYU-Langone Med Center, 550 First Avenue, 10016, NY

5- Department of Experimental, Diagnostic and Specialty Medicine, University of Bologna, Via Massarenti 9, 40138 Bologna

6- Center for Applied Biomedical Research, University of Bologna, Via Massarenti 9, 40138 Bologna

7- corresponding author: paola.bellosta@unitn.it

* equally contributed

running title NOC1, nucleolus and growth

\section{summary statement}

NOC1 is a nucleolar protein necessary for the general control of protein synthesis and cellular growth. In vivo NOC1 expression is required for the correct development of the animal. 


\begin{abstract}
NOC1 is a nucleolar protein necessary in yeast for a correct transport of the large ribosomal subunit and ribosome biogenesis. Here we shown that ubiquitous downregulation of NOC1 in Drosophila results in defective polysome formation and decreases the rate of protein synthesis. Reduction of NOC1 is detrimental for animal growth and adequate expression of NOC1 in organs, such as the prothoracic gland and in the fat body is necessary for proper organ function. In the imaginal discs downregulation of NOC1 results in small cells that die by apoptosis. This event is rescued in $\mathrm{M} /+$ background suggesting that cells with reduced of NOC1 are outcompeted by wild type cells because of their reduced protein synthesis. NOC1 downregulation induces the upregulation of the pro-apoptotic eiger-JNK signaling pathway, that results in the activation of DILP8 compensatory mechanism. Our results demonstrate that NOC1 in Drosophila plays an important role in the regulation of protein synthesis and cell survival, linking its function in the nucleolus to the control of animal growth and development.
\end{abstract}

Keywords 3-6: Drosophila, NOC1, cell competition, apoptosis, eiger, DILP8

\title{
Introduction
}

The NOCs are a large family of nucleolar proteins that play a critical role in the control of protein synthesis and ribo-biogenesis in yeast and plants (Edskes et al., 1998; Li et al., 2009). NOC1, NOC2 and NOC3 are necessary for the correct transport and assembly of the rRNA complexes and maturation of the ribosomal subunits (Dlakic and Tollervey, 2004; Hierlmeier et al., 2013). These proteins are characterized by their ability to form heterodimers via an amino acid stretch, called the NOC-domain, that is present in many of the members of this family (Hierlmeier et al., 2013; Milkereit et al., 2001). NOCs are highly conserved in all eukaryotes and studies in yeast $S$. cerevisiae reveal that Noc1, also called MAK21, forms heterodimers with Noc2 that are necessary for the transport and maturation of the pre-ribosomal $60 \mathrm{~S}$ subunit, while Noc3, bound to Noc2, forms complexes that are required for the transport of the $60 \mathrm{~S}$ subunit into the cytoplasm. Noc1, 2 and 3 do not complement with each other, and their roles are essential for life, as mutations in 
$S$ cerevisiae and in Arabidopsis, where NOC1 is also called Slow walker2, affects growth and viability (Edskes et al., 1998; Li et al., 2009; Milkereit et al., 2001).

In Drosophila, growth occurs during larval development where the proper increase in cell mass and animal size is highly dependent on efficient ribosome biogenesis (Texada et al., 2020). Mutations in genes that regulate this process, like those encoding for ribosomal proteins of the Minute family (Marygold et al., 2007; SaeboeLarssen et al., 1998) or components of the nucleolus like Nop60b/Dyskerin (Tortoriello et al., 2010) and Nopp140 (Baral et al., 2020), present common defects, including a delay in development and reduced body size. Similar phenotypes have also been described for mutations in genes that control rRNA synthesis such as Rpl135 subunit of the RNA-Pol-I complex (Grewal et al., 2005), and for diminutive (dm) the gene encoding for MYC (Johnston et al., 1999), a known master regulator of ribosome biogenesis both in Drosophila and in vertebrates (Barna et al., 2008; Destefanis et al., 2020; Grewal et al., 2005; van Riggelen et al., 2010).

Larval growth is also regulated by hormones and growth factors released by nonautonomous signals and controlled by the fat body that works as sensor to coordinate the release of the Drosophila insulin-like peptides DILPs (DILP2, 3 and 5) from the Insulin Producing Cells (IPCs) in the brain in response to nutrient availability (Geminard et al., 2009; Koyama et al., 2020; Maniere et al., 2020). This process is also coordinated by peaks of ecdysone released at specific times during development to control molting and metamorphosis (Nijhout et al., 2014). If animals are in distress or if their organs are damaged, the level of ecdysone, produced by the prothoracic gland (PG) is reduced by DILP8, a peptide secreted by the damaged cells to activate the Leucine-rich repeat, containing the $\mathrm{G}$ protein-coupled receptor-3 (Lgr3) in the brain that blocks ecdysone synthesis (Colombani et al., 2015; Garelli et al., 2015; Vallejo et al., 2015). Thus DILP8, by decreasing the rate of animal development, allows regeneration of the damaged tissue, a process that has been conserved during evolution to control and assure correct organ growth and animal bilateral symmetry (Boulan and Leopold, 2021). DILP8 upregulation is induced by signaling produced by the dying cells, and in turn its activation has been associated with multiple pathways including p53, Xrp1 and the JNK/eiger/TNF $\alpha$ signaling 
pathways (Akai et al., 2021; Boulan et al., 2019; Ji et al., 2019; Lee et al., 2018; Sanchez et al., 2019) through mechanisms still not completely understood.

In Drosophila, the orthologues of yeast Noc1, Noc2 and Noc3 are annotated as CG7839, CG9246 and CG1234 respectively. In this study we demonstrate that these genes, hereinafter called NOC1, NOC2 and NOC3, play a central role in the control of cellular and animal growth; and their reduction is detrimental for animal development. In particular we show for NOC1 that while its overexpression increases polysomal abundance and is dispensable in differentiated tissues, its ubiquitous reduction results in reduced protein synthesis and defective ribosomal biogenesis. In line with these results, reducing NOC1 in different organs compromises their activity. Moreover, in cells of the imaginal disc, reduction of NOC1 induces apoptosis that is rescued in a Minute/+ background suggesting that NOC1 activity may have a role in the control of cell competition. NOC1-RNAi dying cells activate the eiger/JNK signaling pathways which results in the upregulation of DILP8 and a delay in animal development. In summary, our results show that NOC1 is a novel nucleolar component that functions in Drosophila to control cell growth and survival, a step forward in understanding new mechanisms that link protein synthesis to animal development.

\section{Results}

\section{Drosophila NOC1 is necessary for animal growth.}

Our transcriptome analysis in cells of the wing imaginal discs identify NOC1, NOC2 and NOC3, as nucleolar genes that present a similar expression pattern as MYC, suggesting that they may contribute to its function. NOCs genes are evolutionary conserved and their amino acid sequences share between 32 to $35 \%$ homology with the human orthologue proteins (Fig. 1A, Fig. S1). Interestingly, network analysis using the STRING database on predicted protein-protein interactions for NOC1/CG7839 uncovers that all three NOC proteins form a hub with other nucleolar proteins which play important roles in ribosomal biogenesis, suggesting that they may work in concert to ensure proper nucleolar function (Fig. 1B, C). 
To investigate the role of NOCs in vivo in Drosophila, we initially evaluated the effects of their expression in the whole larvae using the ubiquitous actin promoter. This analysis shows that overexpression of NOC1 leads to small larvae (Fig. 1D, E) that reach pupariation, but fail to become adults (Table 1). On the contrary, NOC1, 2 or 3 downregulation of the respective mRNAs significantly reduces larval size (Fig. $1 \mathrm{D}, \mathrm{E})$ resulting in lethality between first and second instars (Table 1). qRT-PCR analysis shows the level of NOC1-mRNA overexpression and the efficiency of NOCs RNA interference (Fig. 1F). We then analyze if the overexpression of NOC1 could complement for the reduction of NOC2 and NOC3 in whole larvae. This analysis shows that NOC1 overexpression was able to fully rescue the lethality only of NOC1-RNAi larvae (Fig. 1A inset, and Fig. S2), suggesting that it exerts a unique function that does not complement for the loss of NOC2 and NOC3.

In yeast, mutations in NOCs genes affect ribosome biogenesis. To thoroughly investigate whether this function is also conserved in Drosophila, we analyzed whether the expression of NOC1 could impact the correct synthesis or production of ribosomal subunits and polysomes. Polysomal profiling from whole larvae, shows that NOC1 overexpression results in a small increase of the $80 \mathrm{~S}$ subunit and in polysomes as compared to the profile in wild type larvae (Fig. 1G-H). On the contrary, reduction of NOC1 causes an overall decrease in ribosomal subunits, ribosomes, polysomes abundance (Fig. 1G-I), and leads to an accumulation of the $40 S$ and 605 subunits (not shown). We then evaluated whether NOC1 overexpression and its downregulation could affect general protein synthesis. To analyze the rate of protein synthesis we performed a WB SUnSET (Surface Sensing of Translation) assay (Deliu et al., 2017) on lysates from larval tissues incubated with the inhibitor of translation puromycin. Differences in the puromycin-labelled peptides is proportional with the changes in translation and is visualized by western blot using anti puromycin antibodies. These experiments show that larvae with a reduced NOC1 level have a significant reduction in the rate of labeled peptides compared with that of wild type animals (Fig $1 \mathrm{~J}-\mathrm{K}$ ). These data indicate that NOC1 is necessary to sustain a proper protein synthesis rate.

Since no functional antibodies are available to localize the endogenous NOC1 protein, we overexpressed an HA tagged form in cells of the wing imaginal disc. This analysis shows that HA-NOC1 colocalize with the nucleolar protein fibrillarin (Fig. 
$1 \mathrm{~L})$ and to large nuclear granules. Western blot analysis using lysates from whole larvae shows that ubiquitous overexpression of HA-NOC1 results in a protein of about $132 \mathrm{KDa}$ (Fig. 1M), in addition, several other bands of lower molecular weight are visualized by HA antibody. These truncated forms may be the result of proteolysis processes induced by toxicity since overexpression of NOC1 at this stage of development starts to be detrimental for the animal (Table 1).

\begin{tabular}{|c|c|c|c|c|c|}
\hline \multicolumn{6}{|c|}{$\begin{array}{r}\text { Table 1: Characterization of NOC expre } \\
\text { promoters }\end{array}$} \\
\hline \multicolumn{2}{|c|}{ Promoter } & transgene & larval stage & pupal stage & adult \\
\hline \multirow{5}{*}{$\begin{array}{l}\text { 중 } \\
\text { 을 } \\
\frac{0}{0} \\
\frac{0}{3}\end{array}$} & \multirow{5}{*}{$\operatorname{actin}^{1}$} & NOC1 OE & vital & vital & - \\
\hline & & NOC1-RNAi & $\begin{array}{l}\text { dead at second instar small } \\
\text { size }\end{array}$ & - & - \\
\hline & & NOC2-RNAi & $\begin{array}{l}\text { dead at second instar small } \\
\text { size }\end{array}$ & - & - \\
\hline & & NOC3-RNAi & $\begin{array}{l}\text { dead at second instar small } \\
\text { size }\end{array}$ & - & - \\
\hline & & $\begin{array}{l}\text { NOC1-RNAI } \\
\text { NOC1 OE }\end{array}$ & $\begin{array}{l}\text { vital total rescue of the } \\
\text { larval size }\end{array}$ & vital & vital \\
\hline \multirow{4}{*}{ 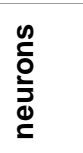 } & \multirow{4}{*}{ elav } & NOC1 OE & vital & vital & vital, no eye defects \\
\hline & & NOC1-RNAi & delayed & lethal & - \\
\hline & & NOC2-RNAi & delayed & lethal & - \\
\hline & & NOC3-RNAi & delayed & lethal & - \\
\hline \multirow{4}{*}{ 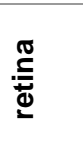 } & \multirow{4}{*}{$G M R^{3}$} & NOC1-RNAi & vital & vital & vital, no eye defects \\
\hline & & NOC1-RNAi & vital & vital & vital, no eye defects \\
\hline & & NOC2-RNAi & vital & vital & vital, no eye defects \\
\hline & & NOC3-RNAi & vital & vital & vital, no eye defects \\
\hline \multirow{4}{*}{ 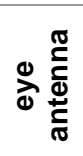 } & \multirow{4}{*}{$\begin{array}{l}\text { tubulin- } \\
\text { ey }>\text { flp }\end{array}$} & NOC1 OE & vital & vital & vital, no eye defects \\
\hline & & NOC1-RNAi & vital & vital & vital, small eye, with defects \\
\hline & & NOC2-RNAi & vital & vital & vital, small eye, with defects \\
\hline & & NOC3-RNAi & vital & vital & vital, small eye, with defects \\
\hline \multirow{4}{*}{ 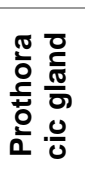 } & \multirow{4}{*}{$P 0206^{5}$} & NOC1 OE & vital & vital & vital, no defects \\
\hline & & NOC1-RNAi & delayed, big larvae & no pupae & - \\
\hline & & NOC2-RNAi & delayed, big larvae & no pupae & - \\
\hline & & NOC3-RNAi & delayed, big larvae & no pupae & - \\
\hline \multirow{8}{*}{ 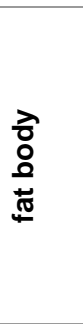 } & \multirow{4}{*}{$\mathrm{Cg}^{6}$} & NOC1 OE & vital & vital & increased body size \\
\hline & & NOC1-RNAi & delayed, partial lethal at L3 & $\begin{array}{l}\text { small pupae mostly } \\
\text { lethal }\end{array}$ & lethal, few escapers * \\
\hline & & NOC2-RNAi & delayed, lethal at L2/L3 & small pupae, lethal & - \\
\hline & & NOC3-RNAi & delayed, lethal at L2/L3 & small pupae, lethal & - \\
\hline & \multirow{4}{*}{$F B$} & NOC1 OE & vital & vital & vital, body size not analyzed \\
\hline & & NOC1-RNAi & delayed, lethal at L3 & small pupae, lethal & - \\
\hline & & NOC2-RNAi & delayed, lethal at L3 & small pupae, lethal & - \\
\hline & & NOC3-RNAi & delayed, lethal at L3 & small pupae, lethal & - \\
\hline \multirow{8}{*}{$\frac{0}{3}$} & \multirow{4}{*}{$\mathrm{MS}_{7} 096$} & NOC1 OE & vital & vital & females: wild-type wings; \\
\hline & & NOC1-RNAi & delayed & vital & vital, crumpled wings \\
\hline & & NOC2-RNAi & delayed & vital & vital, crumpled wings \\
\hline & & NOC3-RNAi & delayed & vital & vital, crumpled wings \\
\hline & \multirow{4}{*}{$\underset{8}{\text { engrailed }}$} & NOC1 OE & vital & vital & vital \\
\hline & & NOC1-RNAi & delayed, lethal ${ }^{* *}$ & - & - \\
\hline & & NOC2-RNAi & delayed, lethal & - & - \\
\hline & & NOC3-RNAi & delayed, lethal & - & - \\
\hline
\end{tabular}

${ }^{1-2}$ see Fig. 1D, ${ }^{3}$ see Fig. 2A-E, ${ }^{4}$ see Fig. 2F-J, ${ }^{5}$ see Fig. $3,{ }^{6}$ see Fig. $4 ;{ }^{7}$ see Fig. $6 ;{ }^{8}$ see Fig $7,{ }^{*}$ few escapers are born with small body size using line on the II Chr. ${ }^{* *}$ few imaginal discs are found using 
the line on the RNAi line on the II-Chr., on the contrary, almost no wing imaginal discs are found using the RNAi line on the III Chr. (see Fig. S3).

Overall, these results suggest that NOC1, NOC2 and NOC3 are essential for a correct animal development. In addition, NOC1 expression in the nucleolus must be maintained at a physiological level since its ubiquitous overexpression is lethal at pupariation, while its reduction strongly impairs ribosome biogenesis and protein synthesis.

\section{NOC1, NOC2 and NOC3 are necessary for the formation of the ommatidia in the compound eye.}

In order to analyze the function of NOCs in tissues with different proliferative characteristics, we use the GMR promoter (Hay et al., 1994) that is expressed from the mid-third instar stage in the differentiated retinal cells of the eye, and the tubulin promoter in combination with eyeless-flippase (Bellosta et al., 2005) to restrain Gal4 expression in the proliferative cells precursors of eye and antenna discs. These data show that downregulating NOC1, 2, or 3, or overexpressing NOC1, under the GMR promoter, does not change the eye morphology (Fig. 2A-E). On the contrary, when using the tubulin promoter downregulation of NOC1, 2 and 3 results in small eyes with fewer and disorganized ommatidia (Fig. 2H-J). Overexpression of NOC1 does not affect the eye morphology (Fig. 2G). These results suggest a role for the NOCs genes in proliferating cells as their reduction late in development in differentiated cells, does not have any effect.

To further characterize the role of NOCs in vivo we evaluated the impact of the downregulation or overexpression of NOC1 in specific organs fundamental for animal physiology and in the control of animal growth.

\section{Reduction of NOC1 in the prothoracic gland controls cell size and affects ecdysone levels inhibiting pupariation.}

Downregulation of the NOC genes in the prothoracic gland (PG), using the P0206Gal4 promoter, results in a strong delay in larval development. These animals reach their third instar stage, do not pupariate and continue to grow for about 20 days 
before dying. Morphological analysis of the PG revealed that at 5 days AEL its size is similar in NOC1-RNAi and in control animals, while in NOC1-RNAi larvae its size is strongly reduced at 12 days AEL (Fig. 3A-C). Cells of the PG are specialized for the production of ecdysone, thus we checked its levels by analyzing the expression of its target Ecdysone-induced protein 74B (E74B) (Valenza et al., 2018). This analysis shows that in NOC1-RNAi animals, E74B mRNA was strongly reduced already at 5 days AEL and at 12 days was further reduced (Fig. 3D). As expected, the overall size of these animals increased over time (Fig. 3F), and so did the size of their fat bodies measured at 12 days AEL (Fig. 3E) and the content of fats (not shown). NOC1 overexpression did not lead to detectable changes in E74B mRNA expression (Fig. 3D), nor to changes in the size of the fat body cells (Fig. 3E).

\section{NOC1 downregulation in the fat body reduces cell size and induces dyslipidemia.}

NOC1, 2, or 3 downregulation in clones in the fat body affects cell morphology and significantly reduces the size of the cells compared to those from wild type animals (Fig. 4A, C-E, F). NOC1 overexpression however does affect cell morphology, but we observed a slight significant reduction in the size of the cells resulting in an overall decrease of the area of the clones (Fig. 4B, F). To investigate the impact of NOC1 in the whole organ, we used the $C g$ (Collagene4a1) promoter (Fig. 4G-R) that is expressed in the fat body and in hemocytes, and the FB promoter that is specific only for the fat cells. Reduction of NOCs, with both promoters, results in larvae with a small body size that display a delay in development and die either at late larval or pupal stages (Table 1). We noticed that the delay in development was less

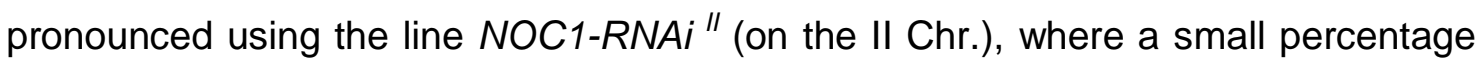
of animals (less than 10\%) hatched as small adults (Fig. 4H, Table 1), possibly because this line is less efficient at reducing endogenous NOC1-mRNA (Fig. S3). Analysis of the fat content, using Nile-red, shows that NOC1-RNAi III larvae (Fig. 4I$\mathrm{L})$ have less lipids compared to wild type animals (Fig. 4M-P). This was particularly visible at the site of the salivary glands (sg) where the fat body associated with them, is almost absent while in wild type animals is quite prominent (Fig. $4 \mathrm{~J}$ and N, arrow). As a response for the reduced capability to store lipids, these animals suffer from dyslipidemia, an inter-organ process usually induced by the failure of fat cells to 
store lipids. Cells with reduced or impaired fat metabolism produces aberrant lipid molecules that non-autonomously induce the accumulation of lipids in other organs (Palm et al., 2012). Indeed, NOC1-RNAi animals unexpectedly accumulated lipids in the wing imaginal discs (Fig. 4M), in the gut (Fig. 4O) and in the brain (Fig. 4P).

The fat body remotely controls the released of Drosophila insulin like peptides (DILP2, 3 and 5) from the Insulin Producing Cells (IPCs). When larva have access to adequate nutrients, the fat body produces signals to trigger the relase of DILPs from the IPCs, while during starvation these signals are absent and DILPs accumulate in the IPCs (Geminard et al., 2009). Analysis of DILP2 expression in the IPCs from animals with reduced NOC1 in the fat body, shows that even in adequate nutrients conditions (FED) DILP2 is retained in these cells (Fig. 4Q-R). This suggest that the signals that control DILPs secretion are lost in the fat cells and reduction of NOC1 mimics animals in starvation where DILPs would ordinarily be retained. On the contrary, overexpression of NOC1 leads to an increase in larval volume at 96 hours AEL. This effect was less significant at 120 hours; nevertheless, adults hatched at a slightly bigger size than control, as shown by analysis of their wing size (Fig. $4 \mathrm{H})$.

\section{NOC1 is necessary for growth and survival of cells in the wing imaginal disc.}

To assess the impact of NOC1 in the growth of epithelial cells we generated random clones co-expressing GFP and analyzed how NOC1, 2 or 3 downregulation or NOC1 overexpression affects their growth, cell size and survival in the wing imaginal discs. NOC1 expressing clones develop to the same size as controls expressing only GFP (Fig. 5A-B, J). On the contrary, downregulation of NOCs caused a significant reduction in the dimension of the clones (Fig. $5 \mathrm{C}-\mathrm{E}, \mathrm{J}$ ). A further analysis using only NOC1-RNAi shows that very few clones were visible when induced at 48 hours AEL suggesting that these cells died and were outcompeted by the neighboring cells (Fig. 5F-G, H). Therefore, we checked whether the expression of the inhibitor of apoptosis p35, along with NOC1 downregulation, could rescue the size of the clones and to visualize NOC1-RNAi cells early before being outcompeted, we induced the clones at 72 hours AEL. With these experimental conditions we were able to visualize small NOC1-RNAi clones, composed of very 
few cells. The size of these clones was overall $15 \%$ of the size of wild type GFP clones, considered 100\% (Fig. 5K-L, I), and co-expression of p35 was able to partially rescue NOC1-RNAi clonal-size up to $60 \%$ (Fig. 5M, I).

These results suggest that cells with reduced NOC1 were subjected to cell competition, a mechanism described for nucleolar components like NOP60b/Dyskerin (Tortoriello et al., 2010) and Nopp140 (Cui and DiMario, 2007), and for Minutes proteins (Akai et al., 2021; Baumgartner et al., 2021; Lee et al., 2018; Nagata and Igaki, 2018; Recasens-Alvarez et al., 2021), where the reduction of biosynthetic activity induces the mutant cells to acquire the characteristics of "loser cells" that are outcompeted by neighboring "winner" wild-type cells and die via apoptosis. To analyze if NOC1 could regulates cell competition by controlling protein synthesis we induced NOC1-RNAi clones in a Minute(3)66D/+ background. These data show that in conditions of systemically reduced protein synthesis NOC1-RNAi clones are partially rescued in size and number (Fig. $5 \mathrm{~N}-\mathrm{O}$ ) acquiring then a growth advantage in condition of partial reduced protein synthesis.

These results suggest a novel role for NOC1 in the control of the biosynthetic activity of the cells as its reduction transforms cells into "losers" that die by apoptosis in a cell competition environment.

\section{Reduction of NOC1 in cells of the imaginal discs induces the pro-apoptotic eiger/JNK signaling and upregulation of DILP8, resulting in a delay in larval development.}

To further characterize NOC1 function in cells of the wing discs, we drove its overexpression and downregulation in the dorsal part of the wing pouch using the MS1096 promoter (Capdevila and Guerrero, 1994). NOC1 overexpression does not affect the size of the disc (Fig. 6C), nor alter the timing of larval development (Fig. $6 \mathrm{G})$. Adults hatch with no defects and with wings at a similar size as those of wild type animals (Fig. 6H). On the contrary, reduction of NOC1 slightly reduces the size of the wing imaginal disc but does not substantially change its morphology (Fig. 6B), adults hatched with defects at the dorsal side of the wings that were also smaller (Fig. 6E, H). The larvae volume was also altered during development, with NOC1RNAi animals being smaller than control (Fig. 6G) and reached pupariation with a 
delay (Fig. 6I). Next, we wondered if this delay was linked to an upregulation of DILP8 in response to the cell death caused by the local depletion of NOC1. Analysis of DILP8 expression shows an upregulation of Dilp8-RNA in discs form NOC1-RNAi animals (Fig. 6J). In agreement with this data, we found a significant increase in dilp8-GFP ${ }^{M 100727}$ reporter in cells with reduced NOC1 expression (Fig. 6K-L). These cells also show an upregulation of the pro-apoptotic gene eiger, visualized using the eiger-GFP ${ }^{f T R G}$ reporter (Fig. 6M-N), that was accompanied with the upregulation of JNK signaling detected using the TRE-GstD1-RFP reporter line (Fig. 60-P). We conclude then that proper NOC1 expression is relevant for the integrity of the epithelial cells of the disc, and its reduction causes nucleolar stress that triggers the pro-apoptotic gene eiger, and ultimately the activation of DILP8/Lgr3 compensatory mechanism. Thus, resulting in the reduction of ecdysone and in the delay in development seen in the NOC1-RNAi animals.

\section{Targeted NOC1 CRISPR-mutation in the posterior compartment of the wing imaginal discs phenocopies NOC1 downregulation induced by RNA interference.}

To develop genomic mutations as useful tools to better study the role of NOC1 and to induce site specific mutations based on the CRISPR-Cas9 system, we used the line $s g R N A^{C G 7839}$ from a library recently developed in Boutros's laboratory to induce site specific mutations based on the CRISPR-Cas9 system (Port et al., 2020). To analyze if the reduction of NOC1 phenocopies the reduction of NOC1 by RNA interference with engrailed-Gal4, we used the promoter hedgehog-Gal4 and drove the expression of Cas 9 in the cells of the posterior compartment of the wing disc together with $\operatorname{sgRNA} A^{C G 7839}$ and GFP. As shown in Fig. 7, driving mutations in NOC1 using the hedgehog/Cas9 system compromises and reduces the development of the posterior compartment of the wing disc (Fig. 7B) with similar results as when NOC1 was reduced using engrailed-Gal4 (Fig. 7D). To compare the efficiency of the two systems, we analyzed the total area of discs and the ratio between the area of the posterior compartment (marked by GFP) and the anterior area, in animals at 120 hours AEL. This analysis shows that in both systems reduction of NOC1 affects the total area of the imaginal discs which is significantly reduced compared to control animals (Fig. 7E, G). The ratio between the posterior and the anterior compartments 
was also reduced with a similar extent in both systems (Fig. 7F, H), suggesting that genetic mutations introduced with the line $s g R N A^{\text {CG7839 }}$ by CRISPR/Cas9 recapitulate the phenotype induced by NOC1-RNAi.

\section{Discussion}

The biogenesis of ribosomes is one of the most complex and energetically demanding processes that takes place primarily in the nucleolus, a distinguishable region of the nucleus where the ribosomal RNA genes (rRNAs) are transcribed into $47 \mathrm{~S}$ pre-RNA and processed to obtain the 18S, 5.8S and $28 \mathrm{~S}$ rRNAs. The 18S, together with the ribosomal proteins, is then directed to form the small ribosomal subunit, while the $5.8 \mathrm{~S}$ and $28 \mathrm{~S}$ rRNAs with the $5 \mathrm{~S}$ and specific riboproteins will from the large 60S subunit (Correll et al., 2019). Many factors have been identified, particularly in yeast, that are necessary for the correct transport and assembly of these complexes, including the NOCs factors (Dlakic and Tollervey, 2004; Hierlmeier et al., 2013).

Here we focused on NOC1, NOC2 and NOC3, as their expression in our transcriptome screens was significantly modulated by MYC expression, a known regulator of ribosome biogenesis (Destefanis et al., 2020). The function of NOC1 in Drosophila is also not redundant, as we found that NOC1 does not compensate for the loss of NOC2 expression (not shown). One possible explanation for the absence of complementarity may relate to NOCs' ability to form heterodimers, which in yeast were shown to be necessary for the transport and maturation of the ribosomal $60 \mathrm{~S}$ subunit, a function that likely requires the formation of unique complexes in which each NOC has a fundamental structural role.

NOC1 contains a CBP (CCAAT binding protein) domain in its amino-acid sequence suggesting that it may have a role as a transcription factor. This hypothesis is corroborated by metadata (CHIP-Seq and genetic screens) in Drosophila, where its expression was associated to promoter regions of genes that control nucleolar activity and ribosomal proteins (Neumuller et al., 2013; Shokri et al., 2019) suggesting that NOC1 may act directly to induce the expression of genes involved in ribosomal biogenesis. A function that may be conserved in humans as shown from a list of selected putative targets for the human homologue CEBPz (or CBF2, CTF2) 
(OMIM-612828) in liver and breast tumors, that includes common nucleolar components and ribosomal proteins (Fig. S4). Our data show that upon overexpression NOC1 co-localizes in the nucleolus with fibrillarin (Fig. 1L) and in large granules in the nucleus. Interestingly, a similar localization in nuclear granules has also been described in HeLa cells for the endogenous CEBPz, which is also a member of the CAAT-Binding protein family. CEBPz can activate transcription to control the Hsp70 promoter (Lum et al., 1990) and it has been shown to play a role in nuclear rRNA processing and 60S transport. Also, the human NOC1/CEBPz proteins contain structural domains which are predicted to mediate protein-protein interactions (Fig. 1A). Overall, these results support the hypothesis that CEBPz may play a similar role to that characterized here in Drosophila however no functional data have been obtained yet.

NOC function is necessary for proper animal development. Ubiquitous reduction of NOC1 evidences a clear defect in growth. These animals were smaller and stopped developing at the second instar, and similar results were observed in animals with reduced levels of NOC2 and NOC3 (Fig. 1A). On the contrary, NOC1 ubiquitous expression allows larvae to reach pupariation at a normal rate but they never eclosed as adults. These results suggest a general function for NOC1 in the nucleolus to control fundamental processes that regulate animal growth that may be linked to defects in translation and in protein synthesis. Indeed, we found that reduction of NOC1 reduces protein synthesis as shown by the robust decrease in the rate of puromycin-labeled peptides evidenced by the WB SUnSET assay (Fig. 1J). Moreover, polysome profiling from these larvae also reveals a reduced ribosome abundance (Fig. 1I) and to an accumulation of ribosomal subunits (Fig. S5), suggesting a defective assembling of the ribosomes in these animals. On the contrary, polysome profiling from NOC1 overexpressing larvae shows a small increase in polysomes (Fig. $1 \mathrm{H}$ ) suggesting that NOC1 plays a role in sustaining the mechanism of translation and protein synthesis.

To have a better indication about NOC1 function in organismal growth, we reduced its expression in organs relevant for Drosophila physiology such as the prothoracic gland (PG), fat body (FB) and the imaginal discs.

Prothoracic gland (PG) 
Reduction of NOC1 in the prothoracic gland (PG) results in a significant decrease in cell size (Fig. 3A-C) and in ecdysone production, as indicated by the levels of its target E74mRNA (Fig. 3D). Consequently, these animals do not develop into pupae and continue to wander as larvae and eventually die at about 20 days AEL. Their larval size increases, and these animals continue to store fats and sugars in cells of the fat body, that are also augmented in size. We previously described the presence of hemocytes (macrophage-like cells) in the fat bodies of these animals at 12 days AEL, whose migration into the fat depends on the amount of ROS (Radical Oxygen Species) likely released by the fat cells in this stressed condition (Valenza et al., 2018). Interestingly, this inter-cellular mechanism recapitulates chronic low-grade inflammation, or Adipocyte Tissue Macrophage (ATM), described in adipose tissue from obese people (Horng and Hotamisligil, 2011).

Fat body

The reduction of NOC1, 2 or 3 levels in clones in the fat body causes cells to be smaller with morphological defects (Fig. 4C-E, F). When NOC1 is reduced in the whole organ using the $\mathrm{Cg}$ promoter, the size of the fat body is reduced (Fig. 4I-P). In Drosophila the fat body controls growth and development by sensing the amino acid concentration in the hemolymph and remotely controls the release of DILP2,3 and 5 from the Insulin Producing Cells in the brain (Andersen et al., 2013; Geminard et al., 2009; Hyun, 2018). At the same time, the fat body stores fats and sugars to be used via the catabolic process of autophagy to survive metamorphosis (Rusten et al., 2004; Scott et al., 2004). When nutrients are reduced, animals delay their development to allow the fat body to accumulate fat and sugars until larvae reach a critical size that ensures them to proceed metamorphosis (Hironaka et al., 2019; Texada et al., 2020). Reducing NOC1 in the fat cells alters their ability to store fats and larvae proceed poorly through development (Fig. 4G), however when NOC1 is reduced by only about $50 \%$ (using an RNAi line that is less efficient S3), some are able to proceed and about $10 \%$ reach their critical size to undergo metamorphosis and eclose as small adults (Fig. 4H).

According to these results, animals with reduced NOC1 in the fat body mimic conditions of low nutrients; indeed, we show DILP2 accumulation in the IPCs. A 
condition described before for animals undergoing starvation or with reduced MYC levels that impaired fat metabolism (Geminard et al., 2009; Parisi et al., 2013). Interestingly, we also observed that Cg-NOC1-RNAi animals abnormally accumulate fats in other organs such in the gut, brain, and in the wing imaginal discs. These findings suggest that these animals exhibit inter organ dyslipidemia, a mechanism of lipid transport active when the fat body is impaired and releases non autonomous signals for the other organs to store fats. A similar process was previously described for mutations in lipoproteins of the APOE family in Drosophila (Palm et al., 2012). Interestingly, this mechanism recapitulates dyslipidemia in humans, when the compromised adipose tissue abnormally releases lipoproteins of the APO family, among others, to induce the accumulation of fats in other organs (Pirillo et al., 2021). While dyslipidemia is most commonly associated with heart and cardiovascular diseases (Rahman et al., 2017), it plays a role also in other organ diseases and pathologies such as diabetic kidney diseases (DKD), non-alcoholic fatty liver disease (NAFLD) and in obesity (Fornoni et al., 2014; Mitrofanova et al., 2021).

Wing imaginal discs

NOC1 function is necessary to control protein synthesis and growth. Indeed, we show in the wing discs with that clones with reduced NOC1 are outcompeted by wild type cells and die by apoptosis (Fig. 5K-M). These defects were partially rescued by reducing protein synthesis in a hypomorphic mutant background with the Minute(3)66D/+ gene (Fig. 5N-O). This suggests that the NOC1-RNAi cells suffer of reduced protein synthesis, also confirmed by SUnSET assay (Fig. 1J). Our data are supporting recent reports that associate mutations in ribosomal proteins to proteotoxic stress and cell competition (Baumgartner et al., 2021; Recasens-Alvarez et al., 2021). Here we propose a novel role for NOC1 in the nucleolus that may act upstream or together with ribosomal proteins to control protein synthesis and cell competition.

The regulation of the final size of an organ is controlled by pathways that integrate systemic and organ-specific signals. Larval development is also controlled by the growth of the imaginal discs, that increase their size by actively proliferating until the animal reaches the larva-pupal transition. Any perturbation of this equilibrium, for 
example by damaging cells of the imaginal discs, results in the activation of an interorgan growth inhibiting mechanism, driven by the secreted factor DILP8. This event coordinates the growth and maturation of the other discs and retards pupariation allowing for the regeneration of the damaged tissues (Colombani et al., 2015; Garelli et al., 2015; Vallejo et al., 2015).

The reduction of NOC1 in a larger compartment of the wing disc, marginally reduces the size of the disc, analyzed at 120 hours AEL (Fig. 6A-C). This was in accord with the reduced size of the larvae measured at the same time of development (Fig. 6G). In addition, these animals show a substantial developmental delay (Fig. 6I), accompanied by an upregulation of DILP8 (Fig. 6L), normally induced by cellular damage. Indeed, we found that cells with reduced NOC1 upregulates eiger (Fig. $6 \mathrm{~N}$ ), a member of the TNF $\alpha$ family and the JNK pathway (Fig. 6P), suggesting that apoptosis in these cells is driven by the eiger-JNK, signaling pathway (Igaki and Miura, 2014). However, many studies are currently undergoing to better define which mechanisms govern cellular damage and we believe that eiger is not the only pathway involved. Indeed, other components have been recently identify that link defects in protein synthesis to apoptosis in cell competition, including the ribosomal protein RpS3 (Baumgartner et al., 2021), RpS12 (Akai et al., 2021; Ji et al., 2019), p53 (Sanchez et al., 2019) and its target Xrp1 (Boulan et al., 2019). At the moment, it is not clear totally how these components interact to each other's in the control of apoptosis and if act non autonomously in the mechanism of cell competition, and

how these pathways induce the release of DILP8 necessary for the coordination of animal growth in Drosophila. Cell competition is a physiological mechanism conserved in many species, we suspect that similar mechanism for inducing apoptosis may also be present in vertebrates for example in the control of cell competition in embryos or during tissue morphogenesis (Claveria et al., 2013; Ellis et al., 2019; Munoz-Martin et al., 2019).

\section{CEBPZ}

Our bioinformatics analysis for the human homologue CEBPz outlines evidence for its role in controlling nucleolar activity, particularly in tumors. Indeed analysis of two 
TCGA datasets from cBio Cancer Genomic Portal (Cerami et al., 2012; Gao et al., 2013) (TGCA: Liver Hepatocellular Carcinoma, GDAC: Firehose and Breast Cancer) (Curtis et al., 2012; Pereira et al., 2016) shows a correlation between altered $C E B P z-m R N A$ expression in liver and breast tumors with the upregulation of nucleolar and ribosomal proteins (S4). The fact that some of these targets, such as Rpl5, Rpl35a, are mutated in patients affected by ribosomapathies (i.e. Diamond Blackfan Anemia -DBA), suggests a potential novel role for $C E B P z$ in controlling nucleolar activity and the insurgence of tumors in patients suffering from these genetic diseases (Mills and Green, 2017; Narla and Ebert, 2010), a connection that, at the moment, is not completely clear.

In summary, our results highlight an important role for the nucleolar NOC1, NOC2 and NOC3 in the control of animal growth and physiology in Drosophila. The reduction of each of the NOCs genes result in similar phenotypes due to the loss of nucleolar integrity and highlight their unique function. In particular we show that NOC1 controls ribosome biogenesis and its reduction causes apoptosis probably as the result of reduced protein synthesis. NOC1 activity is relevant for cell growth and its reduction results in cell competition, a physiological process identified in Drosophila and conserved in vertebrates, where it is involved in regulating the fitness of cells (Claveria and Torres, 2016), but when dis-regulated promotes the insurgence of cancer (Vishwakarma and Piddini, 2020).

\section{Materials and Methods}

\section{Drosophila husbandry and lines}

Animals were raised at low density in small vials containing standard fly food, composed of $9 \mathrm{~g} / \mathrm{L}$ agar, $75 \mathrm{~g} / \mathrm{L}$ corn flour, $50 \mathrm{~g} / \mathrm{L}$ fresh yeast, $30 \mathrm{~g} / \mathrm{L}$ yeast extract, 50 $\mathrm{g} / \mathrm{L}$ white sugar and $30 \mathrm{~mL} / \mathrm{L}$ molasses, along with nipagine (in ethanol) and propionic acid. The crosses and flies used for the experiments are kept at $25^{\circ} \mathrm{C}$, unless otherwise stated.

The following fly lines were used: GMR-Gal4 (Parisi et al., 2011), tub>y+>Gal4; eyflp (Bellosta et al., 2005), P0206-GFP-Gal4 (Valenza et al., 2018), the fat body specific promoter FB-Gal4 (kind gift from Ines Anderl, University of Tampere, 
Finland,) rotund-Gal4 (kind gift from Hugo Stocker, ETH Zurich, CH), actinGal4,UAS-GFP/Gla,Bla (kind gift from Daniela Grifoni University of I' Aquila, IT), yw; Actin>CD2>Gal4,GFP/TM6b (kind gift from Bruce Edgar, University of Boulder, CO), MS1096-Gal4 (kind gift from Erika Bach, NYU, USA), Minute(3)66D/+ (SaeboeLarssen et al., 1997), engrailed-Gal4,GFP and actin-Gal4, GFP; tub-Gal80ts from the lab. The following stocks were obtained from the Bloomington Drosophila Stock Center: Cg-Gal4.A2 (B7011), elav-Gal4 (B458), UAS-CG7839-RNAi (B25992), UASCG9246-RNAi (B50907), UAS-CG1234-RNAi (B618720), dilp8-GFPMI00727 (B33079); and from the Vienna Drosophila Resource Center: UAS-CG7839-RNAi (v12691), and isogenic $w^{1118}$ (v60000) eiger-GFP-2XTY1-SGFP-V5-preTEV-BLRP$3 X F L A G$ (v318615); and the line UAS-CG7839-3xHA was obtained from FlyORF (ZH) (F001775).

\section{Measurement of larval length and volume}

Larvae at the indicated stage of development and genotypes were anesthetized using freezing cold temperature, and pictures were taken using a Leica MZ16F stereomicroscope. Width and length were measured using a grid and volume was calculated by applying the formula in (Parisi et al., 2013).

\section{Quantitative rRT-PCR}

RNA extraction was performed using the RNeasy Mini Kit (Qiagen), following the manufacturer instructions. The isolated RNA was quantified with the Nanodrop2000. $1000 \mathrm{ng}$ of total RNA were retrotranscribed into complementary DNA (cDNA) using the SuperScript IV VILO Master Mix (Invitrogen). The obtained cDNA was used for qRT-PCR using the SYBR Green PCR Kit (Qiagen). The assays were performed on a BioRad CFX96 machine and the analysis were done using Bio-Rad CFX Manager software. Transcript abundance was normalized using actin5c. The list of the primers is available upon request. List of the primers used in Fig. S6.

\section{Dissection and immunofluorescence}


Larvae were collected at the third instar stage, dissected in 1x phosphate-buffered saline (PBS), and fixed for 30 minutes in 4\% paraformaldehyde (PFA) at room temperature $(\mathrm{RT})$. After 15 minutes of tissue permeabilization with $0.3 \%$ Triton $\mathrm{X}$ 100, samples were washed in PBS-0.04\% Tween20 (PBST) and blocked in 1\% bovine serum albumin (BSA) for 1 hour at RT. Samples were incubated overnight at $4^{\circ} \mathrm{C}$ with primary antibodies in $1 \% \mathrm{BSA}$ and, after washing, with Alexa-Fluorconjugated secondary antibodies 1:2000 in BSA. During washing in PBST nuclei were stained with Hoechst. Imaginal discs were dissected from the carcasses and mounted on slides with Vectashield. Imagines were acquired using a Leica SP8 confocal microscope and assembled using Photoshop2020 from Creative Clouds. Secondary antibodies used: Moab rat anti-HA antibodies 1:1000 (Roche 3f10) and Moab anti-fibrillarin (ABCAM ab4566), anti WG 1:100 (DSHB 4D4).

\section{Western blot}

Proteins were extracted from third instar larvae collected in 250 ul of lysis buffer (50 mM Hepes pH 7.4, $250 \mathrm{mM} \mathrm{NaCl}, 1 \mathrm{mM}$ EDTA, 1.5\% Triton X-100) containing a cocktail of phosphatases and proteases inhibitors (Roche). Samples were run on a SDS-polyacrylamide gel and then transferred to a nitrocellulose membrane. After blocking with $5 \%$ non-fat milk in TBS-Tween, membranes were incubated with primary antibodies against puromycin (1:000, clone 12D10 MABE343, Merk)), supernatant mouse anti HA, and actin (1:200, Developmental Studies Hybridoma Bank, 224-236-1), followed by incubation with secondary anti-mouse or rabbit (1:2000 Santa Cruz Biotechnology), and signal was detected using ECL LiteAblot Plus (Euroclone) and the UVITec Alliance LD2.

\section{WB SUnSET assay}

UAS-NOC1-RNAi was expressed ubiquitously in whole larvae using the actin-Gal4 coupled with tubulin-Gal80 temp sensitive allele to avoid early lethality. Crosses were kept at $18^{\circ} \mathrm{C}$ and when larvae reached second instar and were switched to $30^{\circ} \mathrm{C}$ for 72 hours prior to dissection. At least seven third-instar larvae for each genotype were dissected in Schneider's medium and then transferred to Eppendorf tubes containing complete medium with $10 \%$ serum plus puromycin at $20 \mu \mathrm{g} / \mathrm{ml}$ 
(Invitrogen, Thermo Fisher Scientific). The samples were incubated for 40 or 60 minutes at room temperature, then recovered in $10 \%$ serum/media without puromycin for 30 minutes at room temperature. After the inverted larvae were snap frozen in liquid nitrogen for subsequent western blot analysis using anti-puromycin primary antibody.

\section{Polysome profiling}

Cytoplasmic lysates were obtained from snap-frozen whole larvae were pulverized using liquid nitrogen. After addition of lysis buffer and centrifugations for removal of the debris, cleared supernatants were loaded on a linear $10 \%-40 \%$ sucrose gradient and ultracentrifuged in a SW41Ti rotor (Beckman) for $1 \mathrm{hr}$ and $30 \mathrm{~min}$ at $270,000 \mathrm{~g}$ at $4^{\circ} \mathrm{C}$ in a Beckman Optima LE-80K Ultracentrifuge. After ultracentrifugation, gradients were fractionated in $1 \mathrm{~mL}$ volume fractions with continuous monitoring of absorbance at $254 \mathrm{~nm}$ using an ISCO UA-6 UV detector.

\section{Generation of inducible flip-out clones and clonal analysis.}

Females, $y w$; Actin>CD2>Gal4-nGFP/TM6b were crossed with males carrying the heat-shock Flippase $y^{122} w$ together with the relative UAS-transgenes. Animals were left laying eggs for 3-4 hours. Heat shock was performed on larvae at 48 or 72 hours after egg laying $(A E L)$ for $15 \mathrm{~min}$ at $37^{\circ} \mathrm{C}$. Larvae were dissected at 96 or at $120 \mathrm{hrs}$ AEL and mounted using MOWIOL. Images of clones expressing nGFP were acquired using a LEICA SP8 confocal microscope. Quantification of the number of GFP cell clones in the wing imaginal discs was calculated from 5 confocal images for every genotype at 40x magnification maintaining constant acquisition parameters. Co-staining with phalloidin-Rhodamine (Invitrogen) and DAPI was necessary in Fig 6 A-E to outline the cell membranes and the nuclei.

\section{Imaging the adult compound eye and wings}


Photographs of eyes of adult female expressing the indicated UAS-transgenes in the retina using the GMR-Gal4 or tub>y+>Gal4 promoters were taken at 8 days after eclosion using a Leica stereomicroscope MZ16F at 4x magnification.

4-day old animals were fixed in a solution of 1:1 glycerol and ethanol. One wing was dissected from at least 10 animals and mounted on a slide in the same fixing solution. Images of each wing were taken using a Zeiss Axio Imager M2 microscope with a 1x magnification. Quantification of the area of each wing was performed on photographs using PhotoshopCS4.

\section{Fat body staining and cell size calculation}

Fat bodies were dissected from larvae at 5 or 12 days AEL fixed in $4 \%$ PFA and counterstaining with Nile Red (Sigma), Phalloidin-488 (Invitrogen) and Hoechst 33258 (Sigma). After washing with PBS, fat bodies were mounted onto slides with DABCO-Mowiol (Sigma-Aldrich) and images were acquired using LeicaSP5-LEICA, the area of adipose cells for each fat body was calculated with ImageJ software. In order to visualize lipids in the whole larvae, animals were dissected and fixed as above, and stained with Nile-Red after extensive washing in PBS. Dissected organs were mounted in DABCO-Mowiol and photographs were taken using a Zeiss Axio M2 Imager light microscope.

\section{CRISPER-Cas9 mutation of Noc1/CG7839 in the posterior compartment of wing imaginal disc}

We target mutations on CG7938 in the posterior compartment of the wing disc by crossing the line v340019 $\mathrm{P}\{\mathrm{ry}[+\mathrm{t} 7.2]=h s F L P\} 12, \mathrm{y}[1] \mathrm{w}\left[{ }^{*}\right] ; \mathrm{P}\{\mathrm{y}[+\mathrm{t} 7.7] \mathrm{w}[+\mathrm{mC}]=\mathrm{UAS}-$ uMCas9\}attP40; P\{Gal4\}hh-Gal4/TM6B. This line carries the Hedgehog (Hh) Gal4 and the UAS-uMCas9 transgenes to spatially limit the transcription of Cas9 in the posterior region of the animal (Port et al., 2020). This line was crossed with that carrying the gRNA for CG7839 (vCFDlib01132) that was previously recombined with UAS-GFP to mark e visualize the cells in the posterior compartment. A line expressing only UAS-GFP was used as control. F1 animals from crosses were dissected at about 90 hours AEL and GFP and imagines of their wing imaginal discs were acquired using a confocal microscope (Leica-SP8). Calculation of the posterior (GFP) and total area of the wing imaginal discs was performed using Adobe 
Photoshop (Creative Cloud). At least 8 animals from each genotype were used for the statistical analysis.

\section{Statistical Analysis}

Student $t$-test analysis and analysis of the variance calculated using One-way ANOVA with Tukey multi-comparisons test were calculated using GraphPadPRISM8. $p$ values are indicated with asterisks ${ }^{*}=p<0.05,{ }^{* *}=p<0.01,{ }^{* *}=p<$ $0.001,{ }^{* * *}=p<0.0001$, respectively.

\section{Acknowledgements}

We thank the Confocal Facility at IFOM and at CIBIO, the Vienna VDRC and Bloomington Stocks Centers and the DSHB for antibodies. Stocks obtained from the Bloomington Drosophila Stock Center (NIH P40OD018537) were used in this study. We apology in advance to any authors whose work has been omitted.

\section{Competing interest}

No competing interest declared

\section{Funding}

This work was supported by the NIH Public Health Service grant from the NIHSC1DK085047 to PB and SZ, MAE PGR00155 to EMP.

\section{Data availability none}

\section{Legends}

Figure 1: NOCs are important for animal growth and survival; particularly NOC1 controls protein synthesis and polysome abundance. (A) Schematic representation of NOC1, NOC2 and NOC3 proteins and their human homologues. NOC1 contains a CBP domain (CCAAT binding domain), in orange, that shares $32 \%$ sequence identity. Brown represents the conserved NOC domain of 45 amino acids found in NOC1 and NOC3, that share $48 \%$ and $38 \%$ identity between the Dm and human proteins respectively. NOC2 shares an overall $36 \%$ of identity between Dm 
and human NOC2p. Black is representing the region of highest homology (48\%). (B) predicted list of the functional partners of NOC1/CG7839 and their graphic representation $(\mathrm{C})$ using the protein-protein interaction networks generated using STRING (Szklarczyk et al., 2019). (D) Photos of larvae at third instar expressing the indicated transgenes under the actin driver, taken at 120 hours after egg laying $(\mathrm{AEL})$. In the inset are pictures of NOC1-RNAi larvae (middle) rescued by the overexpression of NOC1 (right); these rescued larvae are significantly smaller than control $w^{1118}$ larvae (left) (Supplementary Fig. 2) but are able to undergo metamorphosis and to develop into adult flies (not shown); the scale bar represents $1 \mathrm{~mm}$. (E) Length (in $\mathrm{mm}$.) of larvae of the relative genotype. (F) qRT-PCR showing the amount of NOC-mRNA from instar larvae at 120 hours AEL; actin5C was used as control. (G-I) Representative sucrose density gradient profiles of ribosome from control larvae $(\mathrm{G})$ or animal over-expressing NOC1 $(\mathrm{H})$ or NOC1-RNAi (I). (J) WB SUnSET western blot analysis in lysates from dissected larvae treated with puromycin for the indicated time. The blot shows the relative changes in protein synthesis using anti puromycin antibodies in control $w^{1118}$ or larvae ubiquitously expressing NOC1-RNAi using the actin-promoter. Actin was used as control loading. (K) Graph representing the quantification of the relative change in the rate of puromycin incorporation, normalized to actin is shown. (L) Confocal photo $(63 x)$ of cells overexpressing $H A-N O C 1$ and nuclear GFP to marks the nuclear membrane in cells of the wing disc using the engrailed promoter. Anti HA-staining (red) shows the localization of HA-NOC1 in granules in the nucleus and a partial overlap with antifibrillarin staining the nucleolus (blue). $(\mathrm{H})$ Western blot from lysates of larvae expressing HA-NOC1 using the actin driver, showing a band of $132 \mathrm{KDa}$, as expected from NOC1 amino acid sequence, and a few bands running at a lower molecular weight recognized by the $\mathrm{HA}$ antibody. Data in (E-F) are representative of three experiments, the asterisks represent the $p$-values from One-way analysis of variance (ANOVA) with Tukey multiple comparison ${ }^{*}=p<0.05,{ }^{* *}=p<0.01,{ }^{* *}=p$ $<0.001$ and ${ }^{* * * *}=p<0.0001$, and the error bars indicate the standard deviations.

Figure 2: NOC1, NOC2 and NOC3 are necessary for the formation of the ommatidia in the compound eye. Photographs of Drosophila-compound eyes (lateral view) from males 4 days after eclosion expressing the indicated transgenes in differentiated cells of the eye (A-E) using the GMR-Gal4, or in the proliferative 
cells of the eyeless compartment using the tubulin>CD2>Gal4; eyeless-flp line that constrains Gal4 under eyeless expression (F-J). Similar data were obtained using females (not shown).

Figure 3: NOC1-RNAi in the prothoracic gland reduces cell size and ecdysone and extends development. (A-C) Confocal images of the ring gland marked with GFP using the P0206-GFP driver line in control larvae $w^{1118}(\mathrm{~A})$ and in animals with reduced NOC1 at 5 days (B) and at 12 days AEL. (C). (D) q-RT-PCR showing the level of $E 74 A-m R N A$, target of ecdysone; $(E)$ analysis of cell size in the fat body from control $w^{1118}$ larvae (black) or NOC1-RNAi (blue) larvae at 5 days and at 12 days AEL (light-blue). (F) Photographs of animals with reduced NOCs expression in the prothoracic gland using the P0206-GFP promoter. Picture represents $w^{1118}$ larvae at 5 days $A E L$ and NOC1-3-RNAi at 12 days AEL. The asterisks in D and $E$ represent the $p$-values from $t$-test Student analysis ${ }^{*}=p<0.05$ and ${ }^{* * *}=p<0.0001$, the error bars indicate the standard deviations.

Figure 4: NOC1 downregulation in the fat body reduces cell size and induces dyslipidemia. (A-E) Confocal images of actin-flip-out clones expressing GFP together with the indicated transgenes visualized in the fat body. Phalloidin was used to mark cell membranes. (F) Quantification of the area of the clones. (G) Analysis of the larval volume, measured at the indicated time of development until pupariation, in animals in which NOC1 was reduced using the $\mathrm{Cg}$ promoter. $(\mathrm{H})$ Analysis of the wing size of 4 days old females of the indicated genotypes. Similar data were obtained using males (not shown). (I-P) Photographs of larval organs stained with Nile red to visualized lipids. Reduction of NOC1-RNAi (I-L) reduces the deposition of fats as compared to control (M-P). The inability of the fat body to accumulate fats in NOC1-RNAi animals activates storage of fats in other organs, visible in the wing imaginal discs (wid) $(\mathrm{M})$, in the gut $(\mathrm{O})$ and in the brain $(\mathrm{N})$, $\mathrm{sg}=$ salivary glands. (Q-R) Confocal images of third instar larval brains showing DILP2 immunostaining in the Insulin Producing Cells (IPCs) cells from control (Q) and NOC1-RNAi (R) animals in feeding conditions. Data in (F, G and $\mathrm{H}$ ) are representative of one of three experiments using ten or more animals for each genotype; the asterisks represent the $p$-values from One-way analysis of variance 
(ANOVA) with Tukey multiple comparison ${ }^{*}=p<0.05,{ }^{* *}=p<0.01,{ }^{* * *}=p<$ 0.001 and ${ }^{* * *}=p<0.0001$, and the error bars indicate the standard deviations.

Figure 5: NOC1 is necessary for the growth and survival of cells in the wing imaginal discs. The defects in NOC1-RNAi cells are rescued by co-expression of p35 and in a Minute(3)66D/+ heterozygous background. (A-E) Confocal images of actin-flip-out clones marked by nuclear GFP co-expressing the indicated transgenes and visualized in the wing imaginal discs. Phalloidin was used to mark the cell membranes (red) and DAPI for the nuclei (blue). (J) Quantification of the clonal size was performed by measuring their area and shown in pixels. At least 15 animals from each genotype were used. (F) Photographs of wing imaginal discs showing actin-flip-out clones expressing GFP alone (G) or co-expressing NOC1RNAi. Clones were induced at 48 hours AEL. $(\mathrm{H})$ Quantification of the number of cells in each clone analyzed at 120 hours AEL. (K-M) Photographs of actin-flip-out clones in wing discs expressing GFP with the inhibitor of caspase p35 (K), with NOC1-RNAi (L) or expressing NOC1-RNAi together with p35 (M). Clones were induced at 72 hrs AEL and the cell number in each clone was quantified at 120 hours AEL. (I) The total number of clones analyzed is indicated in parenthesis: $w^{1118}$ + p35 (72, [1.0]), NOC1-RNAi alone (66, [0.15]), NOC1-RNAi + p35 (81, [0.60]). The number in square brackets represents the relative size of the clones compared to that from control, considered equal to 1. (N-O) Analysis of cell number and size or NOC1-RNAi clones induced in wild-type and in a Minute(3)66D/+ background. (N) Quantification of the number of clones in each disc. (O) Analysis of clonal size. These results show that the growth defect of NOC1-RNAi cells is partially rescued when clones are grown with a reduced growth rate using the Minute(3)66D/+ background. The asterisks in $\mathrm{H}, \mathrm{I}, \mathrm{J}, \mathrm{N}$ and $\mathrm{O}$ represent the $p$-values from One-way analysis of variance (ANOVA) with Tukey multiple comparison ${ }^{*}=p<0.05,{ }^{* *}=p<$ $0.01,^{* * *}=p<0.001$ and ${ }^{* * *}=p<0.0001$, and the error bars indicate the standard deviations. Hoechst is used for staining the nuclei.

Figure 6: Reduction of NOC1 activates the eiger-Dilp8 axis inducing developmental delay. Confocal images of wing imaginal discs $(A-C)$, or photos of adult wings (D-F) expressing the indicated transgenes using the MS1096 wingdriver. (G) Larval volume of animals expressing the indicated transgenes using the 
MS1096-driver, measured at the indicated time after egg laying (AEL) until pupariation. $(\mathrm{H})$ Quantification of the size of the wings from adult animals of the indicated genotypes at 4 days after eclosion. Data are expressed as \% from control $w^{1118}$. (I) Curves representing the \% of pupariation in animals of the indicated genotypes. A delay in pupariation is visible in animals where NOC1-RNAi was reduced in the wing pouch. Data are expressed as \% of pupariation over the total number of pupae of the same genotype for each genotype over time, SD is the average of three independent experiments. (J) q-RT-PCR showing the level of Dilp8$m R N A$ in wing imaginal discs overexpressing NOC1 or NOC1-RNAi using the MS1096 promoter, Actin5C mRNA was used as control. (K-P) Confocal images of wing imaginal discs of third instar from $w^{1118}$ and NOC1-RNAi larvae expressing dilp8-GFP (K-L) or eiger-GFP (M-N) proteins, using the rotund-driver or expressing the TRE-GstD1-RFP as reporter for JNK activation using the MS1096 promoter (O$P)$. (G, $\mathrm{HI}$ and $\mathrm{J}$ ) are representative of one of three experiments using ten or more animals for each genotype, the asterisks represent the $p$-values from One-way analysis of variance (ANOVA) with Tukey multiple comparison ${ }^{*}=p<0.05,{ }^{* *}=p<$ $0.01,^{* * *}=p<0.001$ and ${ }^{* * * *}=p<0.0001$, and the error bars indicate the standard deviations. MODEL: The function of nucleolar NOC1 is important for the control of growth and cell survival. Indeed, reduction of NOC1 decreases protein synthesis (showed by SUnSET and with the rescue of defective growth in a Minute/t background), and causes a cellular stress resulting in apoptosis and the upregulation of the pro-apoptotic eiger and JNK pathway. Cells of the wing discs respond with the activation of the DILP8-compensatory mechanism that by reducing ecdysone delays animal development.

Figure 7: Targeted NOC1 CRISPR-mutation in the posterior compartment of the wing imaginal discs, phenocopies the effect of NOC1 downregulation using RNAi interference. (A-D) Confocal images of wing imaginal discs from animals at 120 hours AEL expressing (A-B) hedgehog-Gal4-GFP (hh-Gal4) together with GFP and the uMcas 9 construct alone (A), or together with the $s g-R N A^{C G 7839}$ (B) to conditionally knockout NOC1 in cells of the posterior compartment of the wing disc, marked with GFP expression. (C-D) engrailed (en)-Gal4 expressing GFP in the posterior compartment alone (C), or co-expressing NOC1-RNAi" (D). (E-H) Quantification of the total area $(\mathrm{E}-\mathrm{G})$ of the wing imaginal discs and of the ratio 
posterior/anterior (F-H) of the compartments from control hh-Gal4-GFP (A) and from hh-Gal4-sg-NOC1-GFP larvae (B); or from control en-Gal4-GFP (C) or coexpressing NOC1-RNAi" (D). Data in $(\mathrm{E}-\mathrm{H})$ are representative of one of three experiments using ten or more animal for each genotype, the asterisks represent the $p$-values from $t$-test Students ${ }^{*}=p<0.05,{ }^{* *}=p<0.01,{ }^{* * *}=p<0.001$ and ${ }^{* * *}=p<$ 0.0001 , and the error bars indicate the standard deviations.

\section{References}

Akai, N., Ohsawa, S., Sando, Y. and Igaki, T. (2021). Epithelial cell-turnover ensures robust coordination of tissue growth in Drosophila ribosomal protein mutants. PLoS Genet 17, e1009300.

Andersen, D. S., Colombani, J. and Leopold, P. (2013). Coordination of organ growth: principles and outstanding questions from the world of insects. Trends Cell Biol 23, 336-344.

Baral, S. S., Lieux, M. E. and DiMario, P. J. (2020). Nucleolar stress in Drosophila neuroblasts, a model for human ribosomopathies. Biology open $\mathbf{9}$.

Barna, M., Pusic, A., Zollo, O., Costa, M., Kondrashov, N., Rego, E., Rao, P. H. and Ruggero, D. (2008). Suppression of Myc oncogenic activity by ribosomal protein haploinsufficiency. Nature 456, 971-975.

Baumgartner, M. E., Dinan, M. P., Langton, P. F., Kucinski, I. and Piddini, E. (2021). Proteotoxic stress is a driver of the loser status and cell competition. Nat Cell Biol 23, 136-146.

Bellosta, P., Hulf, T., Balla Diop, S., Usseglio, F., Pradel, J., Aragnol, D. and Gallant, P. (2005). Myc interacts genetically with Tip48/Reptin and Tip49/Pontin to control growth and proliferation during Drosophila development. Proc Natl Acad Sci U S A 102, 11799-11804.

Boulan, L., Andersen, D., Colombani, J., Boone, E. and Leopold, P. (2019). Inter-Organ Growth Coordination Is Mediated by the Xrp1-Dilp8 Axis in Drosophila. Dev Cell 49, 811-818 e814.

Boulan, L. and Leopold, P. (2021). What determines organ size during development and regeneration? Development 148.

Capdevila, J. and Guerrero, I. (1994). Targeted expression of the signaling molecule decapentaplegic induces pattern duplications and growth alterations in Drosophila wings. EMBO J 13, 4459-4468.

Cerami, E., Gao, J., Dogrusoz, U., Gross, B. E., Sumer, S. O., Aksoy, B. A., Jacobsen, A., Byrne, C. J., Heuer, M. L., Larsson, E., et al. (2012). The cBio cancer genomics portal: an open platform for exploring multidimensional cancer genomics data. Cancer Discov 2, 401-404.

Claveria, C., Giovinazzo, G., Sierra, R. and Torres, M. (2013). Myc-driven endogenous cell competition in the early mammalian embryo. Nature 500, 3944.

Claveria, C. and Torres, M. (2016). Cell Competition: Mechanisms and Physiological Roles. Annu Rev Cell Dev Biol 32, 411-439.

Colombani, J., Andersen, D. S., Boulan, L., Boone, E., Romero, N., Virolle, V., Texada, M. and Leopold, P. (2015). Drosophila Lgr3 Couples Organ Growth 
with Maturation and Ensures Developmental Stability. Curr Biol 25, 27232729.

Correll, C. C., Bartek, J. and Dundr, M. (2019). The Nucleolus: A Multiphase Condensate Balancing Ribosome Synthesis and Translational Capacity in Health, Aging and Ribosomopathies. Cells 8.

Cui, Z. and DiMario, P. J. (2007). RNAi knockdown of Nopp140 induces Minute-like phenotypes in Drosophila. Mol Biol Cell 18, 2179-2191.

Curtis, C., Shah, S. P., Chin, S. F., Turashvili, G., Rueda, O. M., Dunning, M. J., Speed, D., Lynch, A. G., Samarajiwa, S., Yuan, Y., et al. (2012). The genomic and transcriptomic architecture of 2,000 breast tumours reveals novel subgroups. Nature 486, 346-352.

Deliu, L. P., Ghosh, A. and Grewal, S. S. (2017). Investigation of protein synthesis in Drosophila larvae using puromycin labelling. Biology open 6, 1229-1234.

Destefanis, F., Manara, V. and Bellosta, P. (2020). Myc as a Regulator of Ribosome Biogenesis and Cell Competition: A Link to Cancer. International journal of molecular sciences 21.

Dlakic, M. and Tollervey, D. (2004). The Noc proteins involved in ribosome synthesis and export contain divergent HEAT repeats. RNA 10, 351-354.

Edskes, H. K., Ohtake, Y. and Wickner, R. B. (1998). Mak21p of Saccharomyces cerevisiae, a homolog of human CAATT-binding protein, is essential for $60 \mathrm{~S}$ ribosomal subunit biogenesis. J Biol Chem 273, 28912-28920.

Ellis, S. J., Gomez, N. C., Levorse, J., Mertz, A. F., Ge, Y. and Fuchs, E. (2019). Distinct modes of cell competition shape mammalian tissue morphogenesis. Nature 569, 497-502.

Fornoni, A., Merscher, S. and Kopp, J. B. (2014). Lipid biology of the podocyte-new perspectives offer new opportunities. Nat Rev Nephrol 10, 379-388.

Gao, J., Aksoy, B. A., Dogrusoz, U., Dresdner, G., Gross, B., Sumer, S. O., Sun, Y., Jacobsen, A., Sinha, R., Larsson, E., et al. (2013). Integrative analysis of complex cancer genomics and clinical profiles using the cBioPortal. Sci Signal 6, pl1.

Garelli, A., Heredia, F., Casimiro, A. P., Macedo, A., Nunes, C., Garcez, M., Dias, A. R., Volonte, Y. A., Uhlmann, T., Caparros, E., et al. (2015). Dilp8 requires the neuronal relaxin receptor Lgr3 to couple growth to developmental timing. Nat Commun 6, 8732.

Geminard, C., Rulifson, E. J. and Leopold, P. (2009). Remote control of insulin secretion by fat cells in Drosophila. Cell Metab 10, 199-207.

Grewal, S. S., Li, L., Orian, A., Eisenman, R. N. and Edgar, B. A. (2005). Mycdependent regulation of ribosomal RNA synthesis during Drosophila development. Nat Cell Biol 7, 295-302.

Hay, B. A., Wolff, T. and Rubin, G. M. (1994). Expression of baculovirus P35 prevents cell death in Drosophila. Development 120, 2121-2129.

Hierlmeier, T., Merl, J., Sauert, M., Perez-Fernandez, J., Schultz, P., Bruckmann, A., Hamperl, S., Ohmayer, U., Rachel, R., Jacob, A., et al. (2013). Rrp5p, Noc1p and Noc2p form a protein module which is part of early large ribosomal subunit precursors in S. cerevisiae. Nucleic Acids Res 41, 1191-1210.

Hironaka, K. I., Fujimoto, K. and Nishimura, T. (2019). Optimal Scaling of Critical Size for Metamorphosis in the Genus Drosophila. iScience 20, 348-358.

Horng, T. and Hotamisligil, G. S. (2011). Linking the inflammasome to obesityrelated disease. Nat Med 17, 164-165. 
Hyun, S. (2018). Body size regulation by maturation steroid hormones: a Drosophila perspective. Front Zool 15, 44.

Igaki, T. and Miura, M. (2014). The Drosophila TNF ortholog Eiger: emerging physiological roles and evolution of the TNF system. Seminars in immunology 26, 267-274.

Ji, Z., Kiparaki, M., Folgado, V., Kumar, A., Blanco, J., Rimesso, G., Chuen, J., Liu, Y., Zheng, D. and Baker, N. E. (2019). Drosophila RpS12 controls translation, growth, and cell competition through Xrp1. PLoS Genet 15, e1008513.

Johnston, L. A., Prober, D. A., Edgar, B. A., Eisenman, R. N. and Gallant, P. (1999). Drosophila myc regulates cellular growth during development. Cell 98, 779-790.

Koyama, T., Texada, M. J., Halberg, K. A. and Rewitz, K. (2020). Metabolism and growth adaptation to environmental conditions in Drosophila. Cell Mol Life Sci 77, 4523-4551.

Lee, C. H., Kiparaki, M., Blanco, J., Folgado, V., Ji, Z., Kumar, A., Rimesso, G. and Baker, N. E. (2018). A Regulatory Response to Ribosomal Protein Mutations Controls Translation, Growth, and Cell Competition. Dev Cell 46, 456-469 e454.

Li, N., Yuan, L., Liu, N., Shi, D., Li, X., Tang, Z., Liu, J., Sundaresan, V. and Yang, W. C. (2009). SLOW WALKER2, a NOC1/MAK21 homologue, is essential for coordinated cell cycle progression during female gametophyte development in Arabidopsis. Plant physiology 151, 1486-1497.

Lum, L. S., Sultzman, L. A., Kaufman, R. J., Linzer, D. I. and Wu, B. J. (1990). A cloned human CCAAT-box-binding factor stimulates transcription from the human hsp70 promoter. Mol Cell Biol 10, 6709-6717.

Maniere, G., Alves, G., Berthelot-Grosjean, M. and Grosjean, Y. (2020). Growth regulation by amino acid transporters in Drosophila larvae. Cell Mol Life Sci 77, 4289-4297.

Marygold, S. J., Roote, J., Reuter, G., Lambertsson, A., Ashburner, M., Millburn, G. H., Harrison, P. M., Yu, Z., Kenmochi, N., Kaufman, T. C., et al. (2007). The ribosomal protein genes and Minute loci of Drosophila melanogaster. Genome Biol 8, R216.

Milkereit, P., Gadal, O., Podtelejnikov, A., Trumtel, S., Gas, N., Petfalski, E., Tollervey, D., Mann, M., Hurt, E. and Tschochner, H. (2001). Maturation and intranuclear transport of pre-ribosomes requires Noc proteins. Cell 105, 499-509.

Mills, E. W. and Green, R. (2017). Ribosomopathies: There's strength in numbers. Science 358.

Mitrofanova, A., Burke, G., Merscher, S. and Fornoni, A. (2021). New insights into renal lipid dysmetabolism in diabetic kidney disease. World $J$ Diabetes 12, 524-540.

Munoz-Martin, N., Sierra, R., Schimmang, T., Villa Del Campo, C. and Torres, M. (2019). Myc is dispensable for cardiomyocyte development but rescues Mycndeficient hearts through functional replacement and cell competition. Development 146.

Nagata, R. and Igaki, T. (2018). Cell competition: Emerging mechanisms to eliminate neighbors. Development, growth \& differentiation 60, 522-530.

Narla, A. and Ebert, B. L. (2010). Ribosomopathies: human disorders of ribosome dysfunction. Blood 115, 3196-3205. 
Neumuller, R. A., Gross, T., Samsonova, A. A., Vinayagam, A., Buckner, M., Founk, K., Hu, Y., Sharifpoor, S., Rosebrock, A. P., Andrews, B., et al. (2013). Conserved regulators of nucleolar size revealed by global phenotypic analyses. Sci Signal 6, ra70.

Nijhout, H. F., Riddiford, L. M., Mirth, C., Shingleton, A. W., Suzuki, Y. and Callier, V. (2014). The developmental control of size in insects. Wiley interdisciplinary reviews. Developmental biology 3, 113-134.

Palm, W., Sampaio, J. L., Brankatschk, M., Carvalho, M., Mahmoud, A., Shevchenko, A. and Eaton, S. (2012). Lipoproteins in Drosophila melanogaster--assembly, function, and influence on tissue lipid composition. PLoS Genet 8, e1002828.

Parisi, F., Riccardo, S., Daniel, M., Saqcena, M., Kundu, N., Pession, A., Grifoni, D., Stocker, H., Tabak, E. and Bellosta, P. (2011). Drosophila insulin and target of rapamycin (TOR) pathways regulate GSK3 beta activity to control Myc stability and determine Myc expression in vivo. BMC Bio/ 9, 65.

Parisi, F., Riccardo, S., Zola, S., Lora, C., Grifoni, D., Brown, L. M. and Bellosta, P. (2013). dMyc expression in the fat body affects DILP2 release and increases the expression of the fat desaturase Desat1 resulting in organismal growth. Dev Biol 379, 64-75.

Pereira, B., Chin, S. F., Rueda, O. M., Vollan, H. K., Provenzano, E., Bardwell, H. A., Pugh, M., Jones, L., Russell, R., Sammut, S. J., et al. (2016). The somatic mutation profiles of 2,433 breast cancers refines their genomic and transcriptomic landscapes. Nat Commun 7, 11479.

Pirillo, A., Casula, M., Olmastroni, E., Norata, G. D. and Catapano, A. L. (2021). Global epidemiology of dyslipidaemias. Nat Rev Cardiol.

Port, F., Strein, C., Stricker, M., Rauscher, B., Heigwer, F., Zhou, J., Beyersdorffer, C., Frei, J., Hess, A., Kern, K., et al. (2020). A large-scale resource for tissue-specific CRISPR mutagenesis in Drosophila. eLife $\mathbf{9}$.

Rahman, M. S., Murphy, A. J. and Woollard, K. J. (2017). Effects of dyslipidaemia on monocyte production and function in cardiovascular disease. Nat Rev Cardiol 14, 387-400.

Recasens-Alvarez, C., Alexandre, C., Kirkpatrick, J., Nojima, H., Huels, D. J., Snijders, A. P. and Vincent, J. P. (2021). Ribosomopathy-associated mutations cause proteotoxic stress that is alleviated by TOR inhibition. Nat Cell Biol 23, 127-135.

Rusten, T. E., Lindmo, K., Juhasz, G., Sass, M., Seglen, P. O., Brech, A. and Stenmark, H. (2004). Programmed autophagy in the Drosophila fat body is induced by ecdysone through regulation of the PI3K pathway. Dev Cell 7, 179-192.

Saeboe-Larssen, S., Lyamouri, M., Merriam, J., Oksvold, M. P. and Lambertsson, A. (1998). Ribosomal protein insufficiency and the minute syndrome in Drosophila: a dose-response relationship. Genetics 148, 1215 1224.

Saeboe-Larssen, S., Urbanczyk Mohebi, B. and Lambertsson, A. (1997). The Drosophila ribosomal protein L14-encoding gene, identified by a novel Minute mutation in a dense cluster of previously undescribed genes in cytogenetic region 66D. Mol Gen Genet 255, 141-151.

Sanchez, J. A., Mesquita, D., Ingaramo, M. C., Ariel, F., Milan, M. and Dekanty, A. (2019). Eiger/TNFalpha-mediated Dilp8 and ROS production coordinate intra-organ growth in Drosophila. PLoS Genet 15, e1008133. 
Scott, R. C., Schuldiner, O. and Neufeld, T. P. (2004). Role and regulation of starvation-induced autophagy in the Drosophila fat body. Dev Cell 7, 167-178.

Shokri, L., Inukai, S., Hafner, A., Weinand, K., Hens, K., Vedenko, A., Gisselbrecht, S. S., Dainese, R., Bischof, J., Furger, E., et al. (2019). A Comprehensive Drosophila melanogaster Transcription Factor Interactome. Cell Rep 27, 955-970 e957.

Szklarczyk, D., Gable, A. L., Lyon, D., Junge, A., Wyder, S., Huerta-Cepas, J., Simonovic, M., Doncheva, N. T., Morris, J. H., Bork, P., et al. (2019). STRING v11: protein-protein association networks with increased coverage, supporting functional discovery in genome-wide experimental datasets. Nucleic Acids Res 47, D607-D613.

Texada, M. J., Koyama, T. and Rewitz, K. (2020). Regulation of Body Size and Growth Control. Genetics 216, 269-313.

Tortoriello, G., de Celis, J. F. and Furia, M. (2010). Linking pseudouridine synthases to growth, development and cell competition. The FEBS journal 277, 3249-3263.

Valenza, A., Bonfanti, C., Pasini, M. E. and Bellosta, P. (2018). Anthocyanins Function as Anti-Inflammatory Agents in a Drosophila Model for Adipose Tissue Macrophage Infiltration. BioMed research international 2018, 6413172.

Vallejo, D. M., Juarez-Carreno, S., Bolivar, J., Morante, J. and Dominguez, M. (2015). A brain circuit that synchronizes growth and maturation revealed through Dilp8 binding to Lgr3. Science 350, aac6767.

van Riggelen, J., Yetil, A. and Felsher, D. W. (2010). MYC as a regulator of ribosome biogenesis and protein synthesis. Nat Rev Cancer 10, 301-309.

Vishwakarma, M. and Piddini, E. (2020). Outcompeting cancer. Nat Rev Cancer 20, 187-198. 


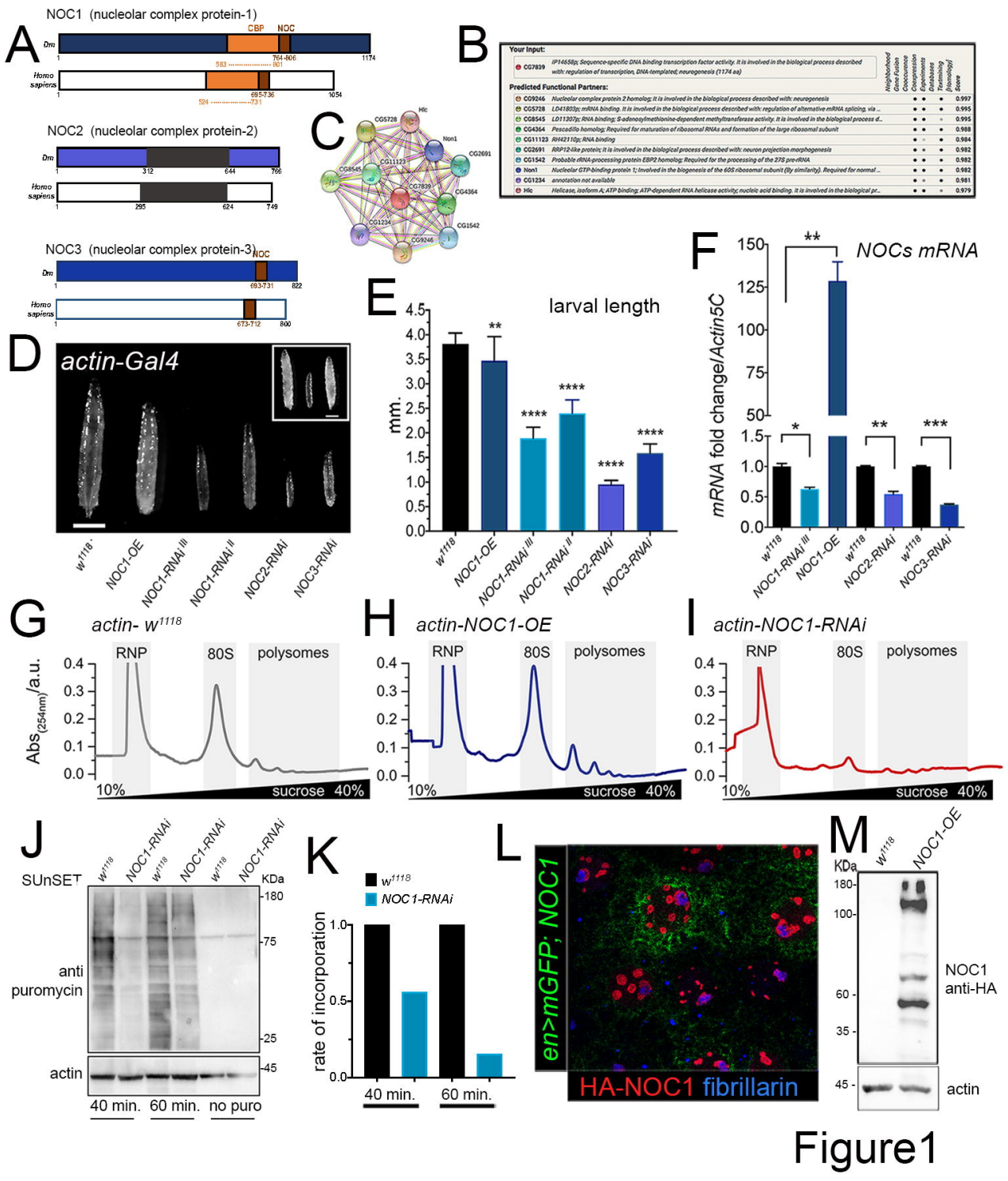




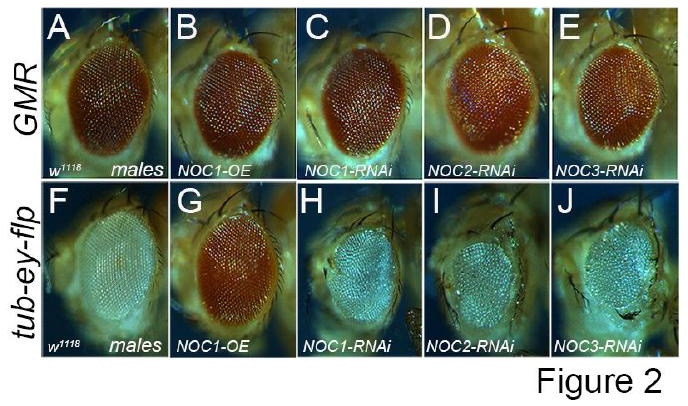




\section{$P 0206>$ GFP}

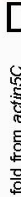

4

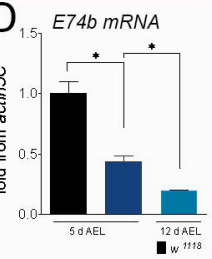

\section{NOC1-RNAi}

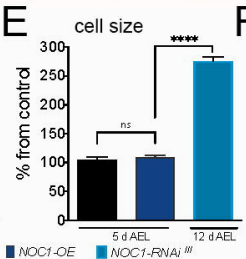

NOC1-RNAi

P0206-GFP

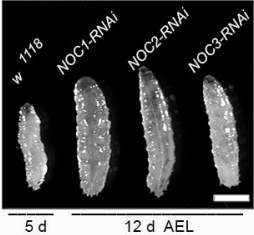

Figure 3 


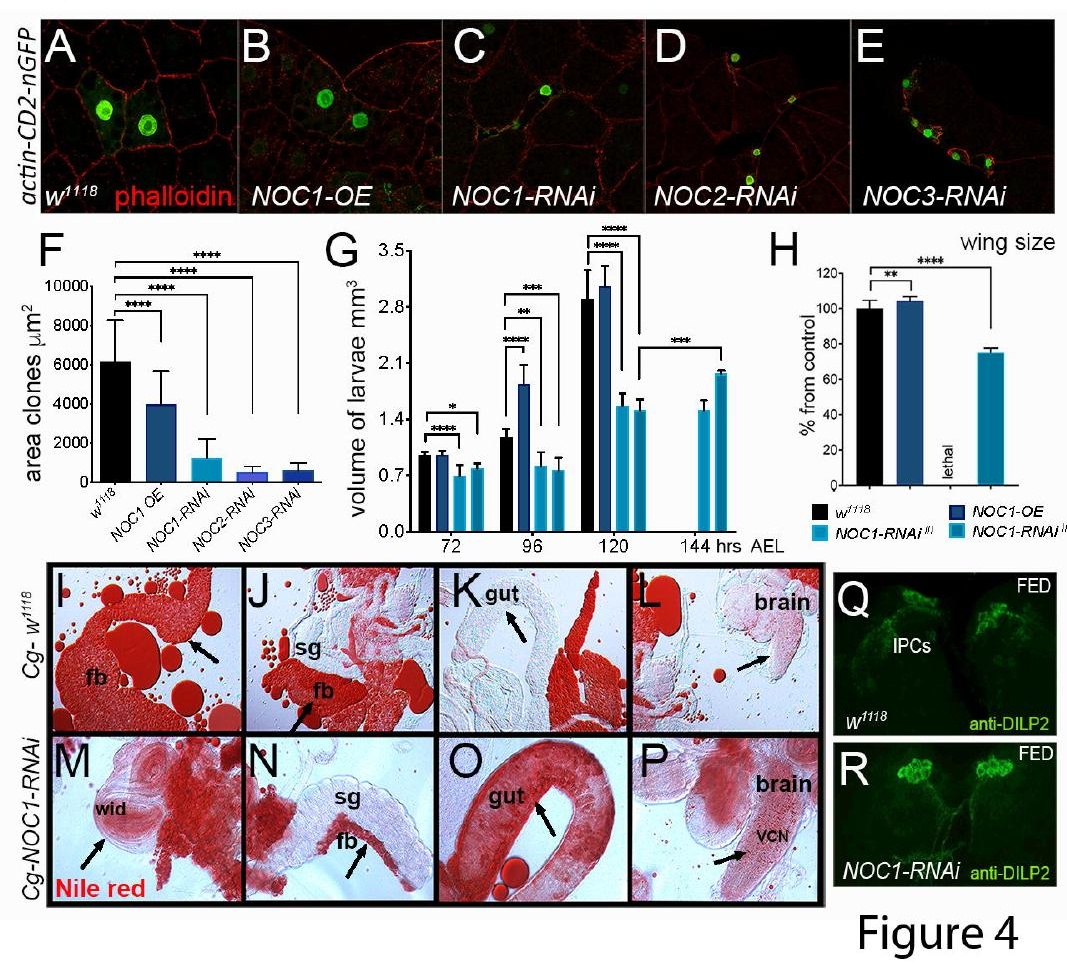




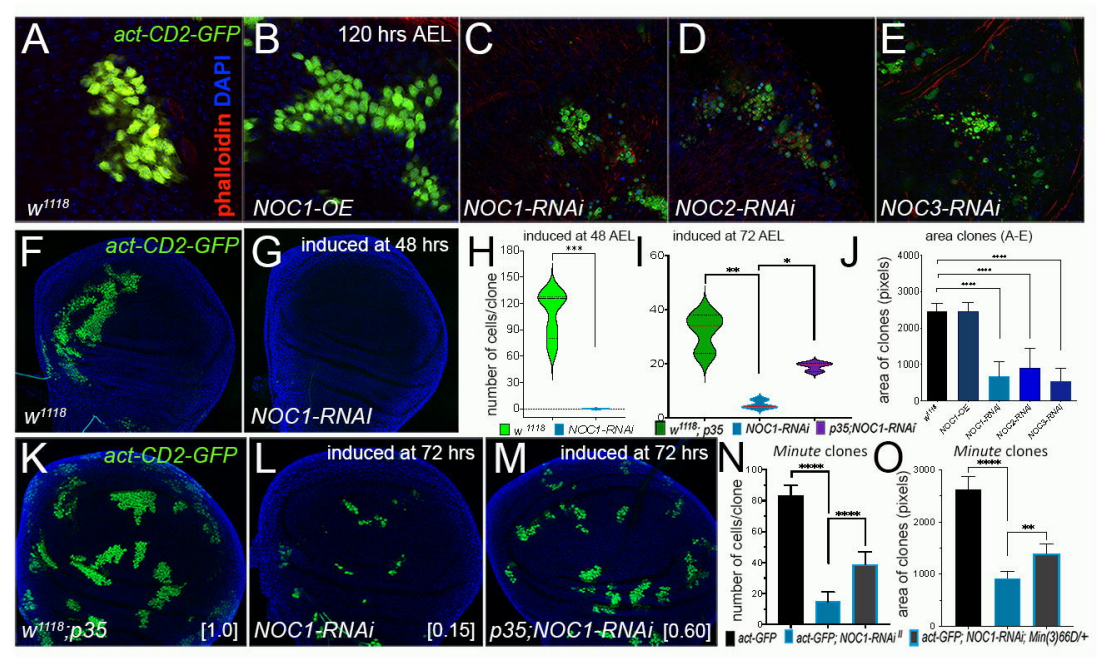

Figure 5 

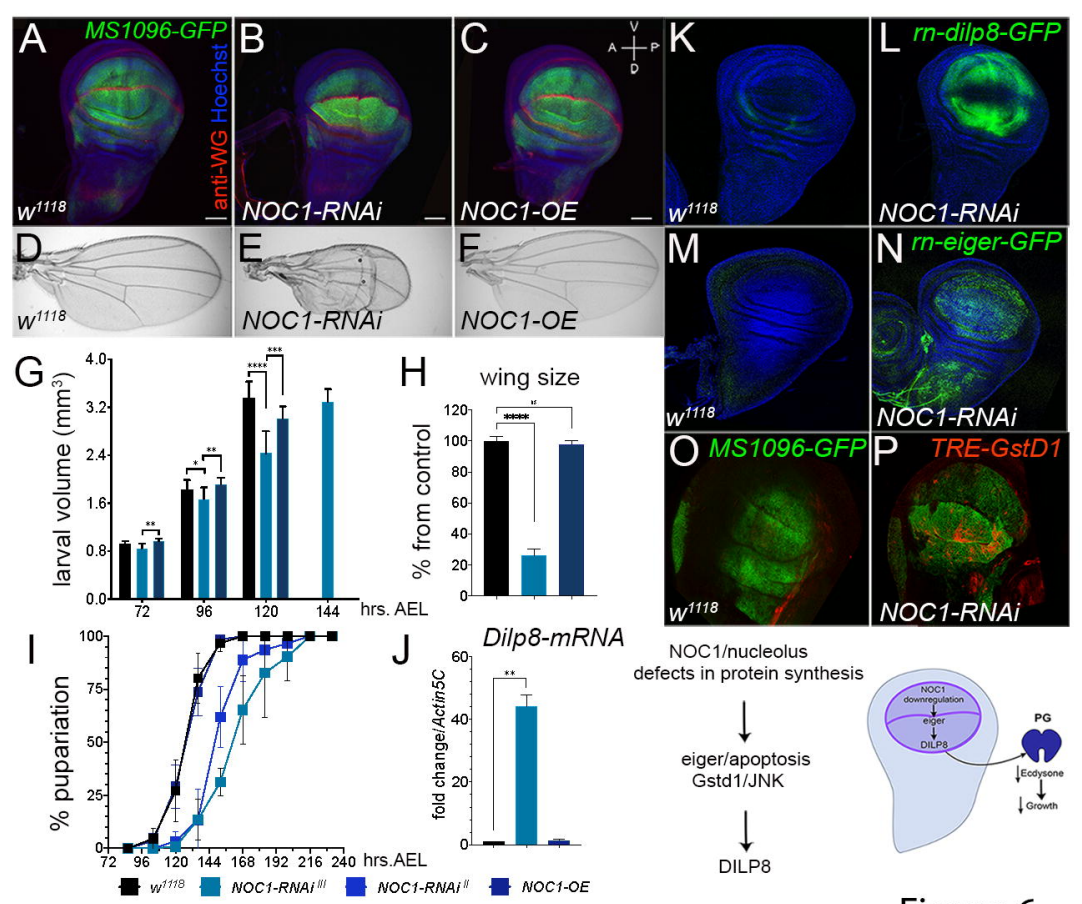

Figure 6 


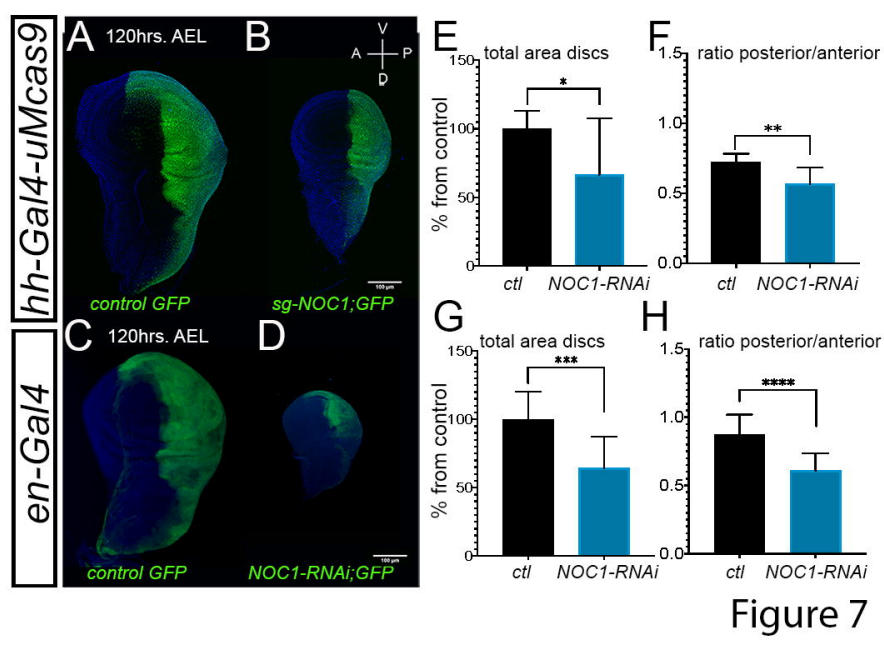

